\title{
Monitoring laser-tissue interaction by non-linear optics
}

\author{
Tsung-Hua Tsai ${ }^{1,2}$, Jin-Ning Lee ${ }^{3}$, Jung-Yi, Chan ${ }^{4,5}$, Jr-Ting Hsu ${ }^{3}$, Hsin-Yuan Tan ${ }^{6}$, Chen-Yuan \\ Dong $^{3}$, Woan-Ruoh Lee ${ }^{2,7}$, Sung-Jan Lin ${ }^{5,8}$ \\ ${ }^{1}$ Department of Dermatology, Far Eastern Memorial Hospital, Taipei, Taiwan. \\ ${ }^{2}$ Graduate Institute of Medical Sciences, Taipei Medical University, Taipei, Taiwan. \\ ${ }^{3}$ Department of Physics, National Taiwan University, Taipei, Taiwan. \\ ${ }^{4}$ Department of Dermatology, Cathay General Hospital, Taipei, Taiwan. \\ ${ }^{5}$ Department of Dermatology, National Taiwan University Hospital and College of Medicine, Taipei, \\ Taiwan. \\ ${ }^{6}$ Department of Ophthalmology, Chang Gung Memorial Hospital, Linkou, Taiwan. \\ ${ }^{7}$ Department of Dermatology, Taipei Medical University Hospital, Taipei, Taiwan. \\ ${ }^{8}$ Institute of Biomedical Engineering, National Taiwan University, Taipei, Taiwan
}

\begin{abstract}
To assess laser therapies in clinical practice, histologic examinations were commonly used. But histologic examinations were invasive and not real-time in nature. In this work, we validate multiphoton microscopy as a useful modality in evaluating laser-tissue reaction in vivo. Multiphoton microscopy based on femtosecond titanium-sapphire laser system were used to evaluate autoflurescence (AF) and second harmonic generation (SHG). Nude mouse skin was irradiated with Erbium:YAG laser at low to high fluence. High resolutional images can be obtained by multiphoton microscopy. At low fluence, Erbium:YAG laser can selectively loosen compact stratum corneum with minimal injury to basal layer. At high fluence, ablated keratinocytes and residual debris can be imaged. The laser thermal effect on dermis could be measured by SHG signals of collagen fibers. SHG decreased as laser fluence increased. Multiphoton microscopy is a useful in-vivo technique in evaluating ablative and thermal effects of Erbium:YAG laser on nude mouse skin.
\end{abstract}

Keywords: laser, multiphoton, fluorescence, second harmonic generation, collagen

Address correspondence to: W.R.L. at wrlee@tmu.edu.tw

$$
\text { S.J.L. at } \underline{\text { drsjlin@ntu.edu.tw }}
$$

Photonic Therapeutics and Diagnostics IV, edited by Nikiforos Kollias, Bernard Choi, Haishan Zeng, Reza S. Malek, Brian Jet-Fei Wong, Justus F. R. Ilgner, Kenton W. Gregory, Guillermo J. Tearney, Henry Hirschberg, Steen J. Madsen, Proc. of SPIE Vol. 6842, 684202, (2008) - 1605-7422/08/\$18 · doi: 10.1117/12.761407 


\section{Introduction}

Various light therapies have been employed in clinical practice. Erbium:YAG laser is widely used in dermatologic surgery, including ablation of skin hyperplasia and tumors, removal of pigmented nevus, and dermabrasion of acne scar or rejuvenation. ${ }^{1-3}$ Er:YAG laser enhanced transdermal drug delievery has also been studied. ${ }^{4-10}$

The results of laser treatment depend largely on the parameters of laser treatment and laser-tissue interaction. Despite the wide application of light therapy, serial tissue reaction and the subsequent tissue remodeling have not been investigated in detail due to the lack of an appropriate real-time noninvasive monitoring tool. Most investigators resort to other more objective methods to assess laser results, including ultrasound and optical coherence tomography. ${ }^{11,} 12$ However, the resolution rates of these modalities are limited. As in many areas of medicine, the post-laser result remains to rely on histopathologic examination in the evaluating the epidermal, dermal, vascular and appendage components of skin. ${ }^{13,14}$ The drawbacks in this procedure are invasive in nature and that the fixation and processing procedures render the studying of real time light-tissue interaction impossible.

The development of non-invasive imaging techniques such as confocal scanning laser microscopy helped to improve the in vivo diagnosis of skin condition. ${ }^{15,16}$ However, multiphoton microscopy has emerged to be another useful technique in the applications of dermatology. The precursor of multiphoton imaging is two-photon fluorescence microscopy (TPFM). ${ }^{17}$ TPFM holds several unique advantages over confocal scanning laser microscopy, such as reduced specimen photobleaching, higher-contrast images and enhanced penetration depth. ${ }^{18}$ In vivo imaging of human skin has been achieved. ${ }^{19}$ In addition to TPFM, the non-linear polarization effect of second-harmonic generation (SHG) has emerged as a viable microscope contrast mechanism for visualization of cell and tissue structure and function. SHG enables direct imaging of anisotropic biological structures possessing large hyperpolarizabilities, such as collagen, microtubules, and muscle myosin. ${ }^{20}$ Recently, SHG has been applied to monitor the thermally induced structural transitions of collagen and to predict heat-induced collagen shrinkage. ${ }^{21-23}$ Combination of multiphoton autofluorescence (AF) and SHG imaging has been applied to evaluate cutaneous photoaging and discriminate basal cell carcinoma from normal dermal stroma. ${ }^{24,25}$

The aims of this study were to investigate the morphological and quantitative change of AF and SHG signals after Erbium:YAG laser and to evaluate the potential of multiphoton microscopy in real-time evaluating the effect of laser-skin interaction

\section{Materials and Methods}

Male nude mice (Balb/c-nu strain, 9 weeks old) was anesthesia by phenobarbitol and was prepared for Erbium:YAG laser irradiation. The nude mouse was adapted to multiphoton microscopy to acquire non-linear optics images. The full-thickness skin from dorsal back was excised and prepared for comparable histopathologic examination. An Erbium:YAG laser (Burane, WaveLight Laser Technologie AG, Germany) was used for treating skin. The laser has a wavelength of $2940 \mathrm{~nm}$ and a pulse duration of $250 \mu \mathrm{s}$. An articulated arm was used to deliver the laser beam onto the skin. We gave each sample one shot. The multiphoton microscope system used in this study was based on a 
diode-pumped solid-state (DPSS) laser (Millennia X, Spectra Physics, Mountain View, Calif.) pumped femtosecond titanium-sapphire laser system (Tsunami, Spectra Physics) as the excitation source. The 780-nm output of the laser system was guided toward a modified commercial upright microscope (E800, Nikon, Japan). Prior to entering the microscope, the excitation source was angularly deflected by an x-y scanning system (Model 6220, Cambridge Technology, Cambridge, Mass). The input of the upright microscope was modified to accommodate a beam expander. The excitation source was beam expanded and reflected toward the focusing objective (Nikon S Fluor 40x, NA 1.30) by a primary dichroic (700DCSPXRUV-3p, Chroma Technology, Rockingham, Vermont). The primary dichroic is a short-pass filter that transmits below the wavelength range of 350 to $700 \mathrm{~nm}$. The power at the sample was $35 \mathrm{~mW}$. The nonlinear AF and SHG signal were generated at the sample focal plane and collected in the epi-illuminated or backscattering geometry by the same focusing objective. After passing through the primary dichroic, the AF and SHG signals were separated into two separate channels where they are detected by independent photomultiplier tubes (R7400P, Hamamatsu, Japan). The AF and SHG signals were separated by a secondary dichroic (435DCXR, Chroma Technology). The secondary dichroic is a long-pass filter that reflects below and transmits above $435 \mathrm{~nm}$. The SHG signal was further filtered by a SHG filter (HQ390/20, Chroma Technology) centered at $390 \mathrm{~nm}$ with a bandwidth of $20 \mathrm{~nm}$ while the AF was detected by a bandpass filter (E700sp-2P-E435lp, ChromaTechnology) for broadband fluorescence detection between 435 and $700 \mathrm{~nm}$. To acquire a global image of the thermally treated specimens at high resolution, an x-y sample positioning stage (H101, Prior Scientific, UK) was used to translate the skin specimen after each imaged frame. An array of overlapping and individually beam scanned images, each with an area of 110 x $110 \mu \mathrm{m}^{2}$ was then assembled. Histologic changes in nude mouse skin were examined after laser irradiation. The adjacent untreated skin was assessed as the control group. Each specimen was fixed in 10\% buffered formaldehyde solution at $\mathrm{pH} 7.4$ for 48 hours. The specimen was cut vertically against the skin surface. Each section was dehydrated using ethanol, embedded in paraffin wax, and stained with hematoxylin and eosin.

\section{Results}

Figure 1 clearly demonstrated the multiphoton imaging of nude mouse skin. Figure 1A revealed surface horny layer. Single corneocytes are visible. Figure 1B to C showed characteristic flattening of keratinocyte as they move from the basal layer toward stratum spinosum. Figure $1 \mathrm{C}$ also showed dermal-epidermal junction with mixed AF signals of basal keratinocytes and SHG signals of collagen fibers. Figure 1D showed SHG signals of collagen fibers in dermis.

Figure 2 shows the representative AF and SHG images of skin irradiated by low fluence laser $\left(1.5 \mathrm{~J} / \mathrm{cm}^{2}\right)$. High intensity of AF signals was noted at the horny layer after laser irradiation (Figure 1A). The corneal layer was ablated by laser. In the spinous layer and basal layer, the keratinocytes were still intact (arrow) but the intensity of AF was decreased (Figure 2B-2C). Collagen fibers were still present at dermis but appeared to aggregate into a thick bundle and SHG intensity decreased (Figure 2D).

Figure 3 shows the images of skin irradiated by high fluence $\left(13 \mathrm{~J} / \mathrm{cm}^{2}\right)$ laser. Most corenocyte and keratinocytes were ablated and only residual crusts were left presented as high AF intensity (Figure 3A-3B). Furthermore, in the basal 
layer, only few basal keratinocytes were left but the intensity of image was low. In the dermis, very few collagens were noted. This is comparable with histopathologic findings (Figure 4). Most corenal layer and epidermal keratinocytes were ablated. The dermal collagen beneath the laser irradiated area was eosinophilic degeneration in contrast to the pink well-formed collagen bundles of the unaffected dermis (Figure 4).
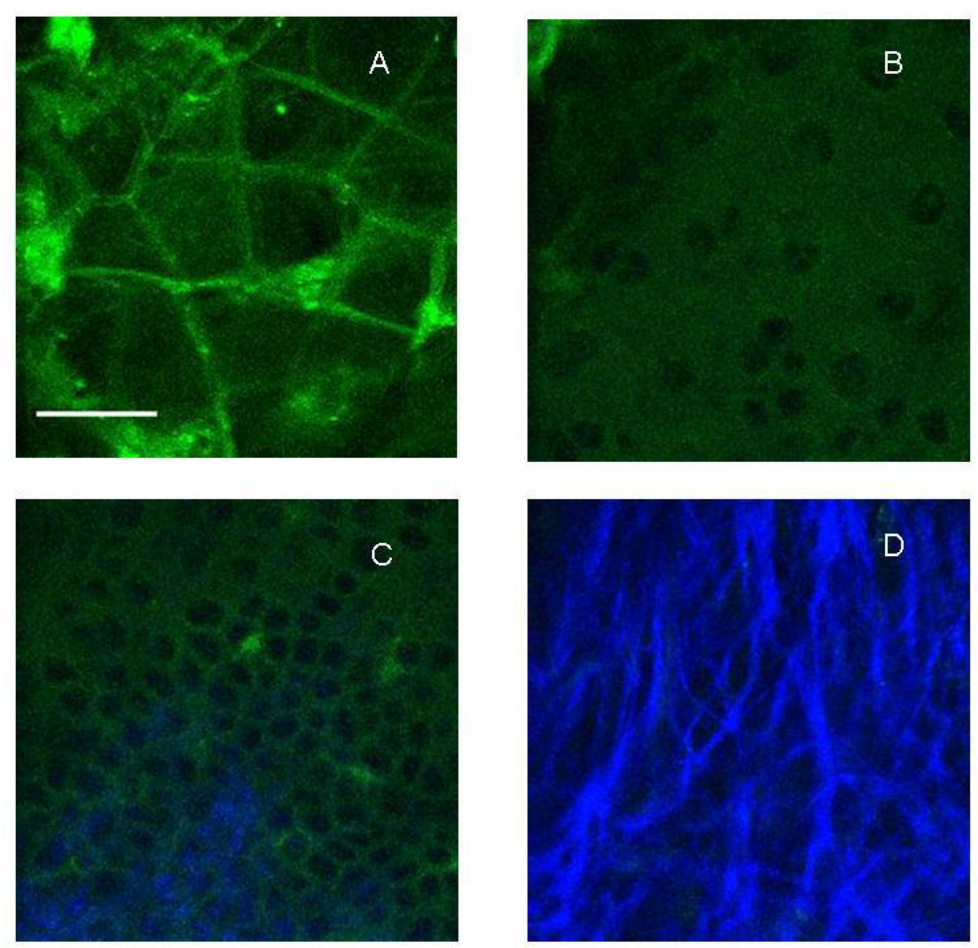

Figure 1. 110 x $110 \mu \mathrm{m}^{2}$ multiphoton combined AF and SHG imaging of normal nude mice skin AF signals are green and SHG signals are blue. (Bar $=20 \mu \mathrm{m})$ 

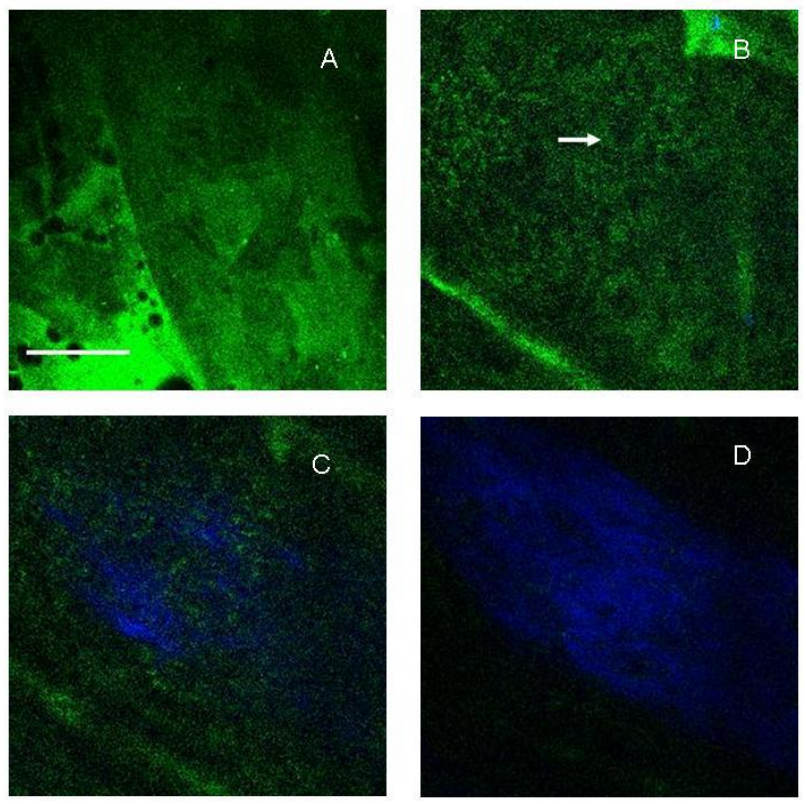

Figure 2. $110 \times 110 \mu \mathrm{m}^{2}$ multiphoton AF and SHG imaging of skin irradiated with Erbium:YAG laser at $1.5 \mathrm{~J} / \mathrm{cm}^{2}$ fluence. Note that no corneal layer was left and some keratinocytes were ablated at upper spinous layer (A). keratinocytes can be seen at lower cspinous layer (B) and basal layer (C), but the AF intensity of keratinocytes diminished (arrow). Numbers of collagen fibers decreased (D). $\mathrm{Bar}=30 \mu \mathrm{m}$
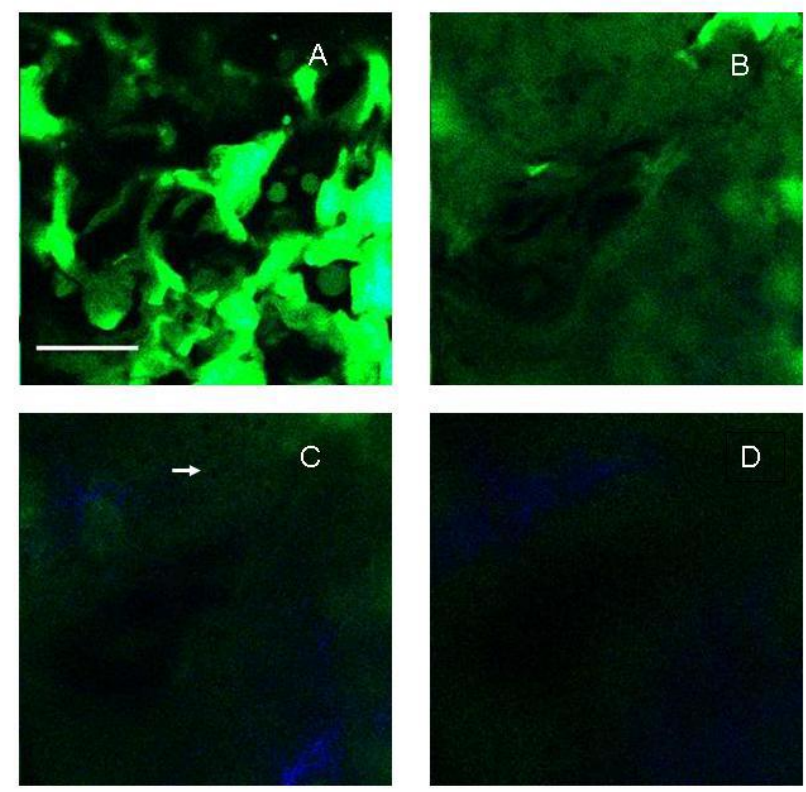

Figure 3. $110 \times 110 \mu \mathrm{m}^{2}$ multiphoton combined AF and SHG imaging of nude mouse skin after Erbium:YAG treatment at 13 $\mathrm{J} / \mathrm{cm}^{2}$ fluecne. Amorphous intense AF was noted after laser irradiation and no corneocytes were visible (A). Also, keratinocytes in the 
spinous layer and basal layer became amorphous and unclear $(B \& C)$. In the dermis, there were very weak SHG signals left without normal collagen bundles (D). Bar=30 $\mu \mathrm{m}$

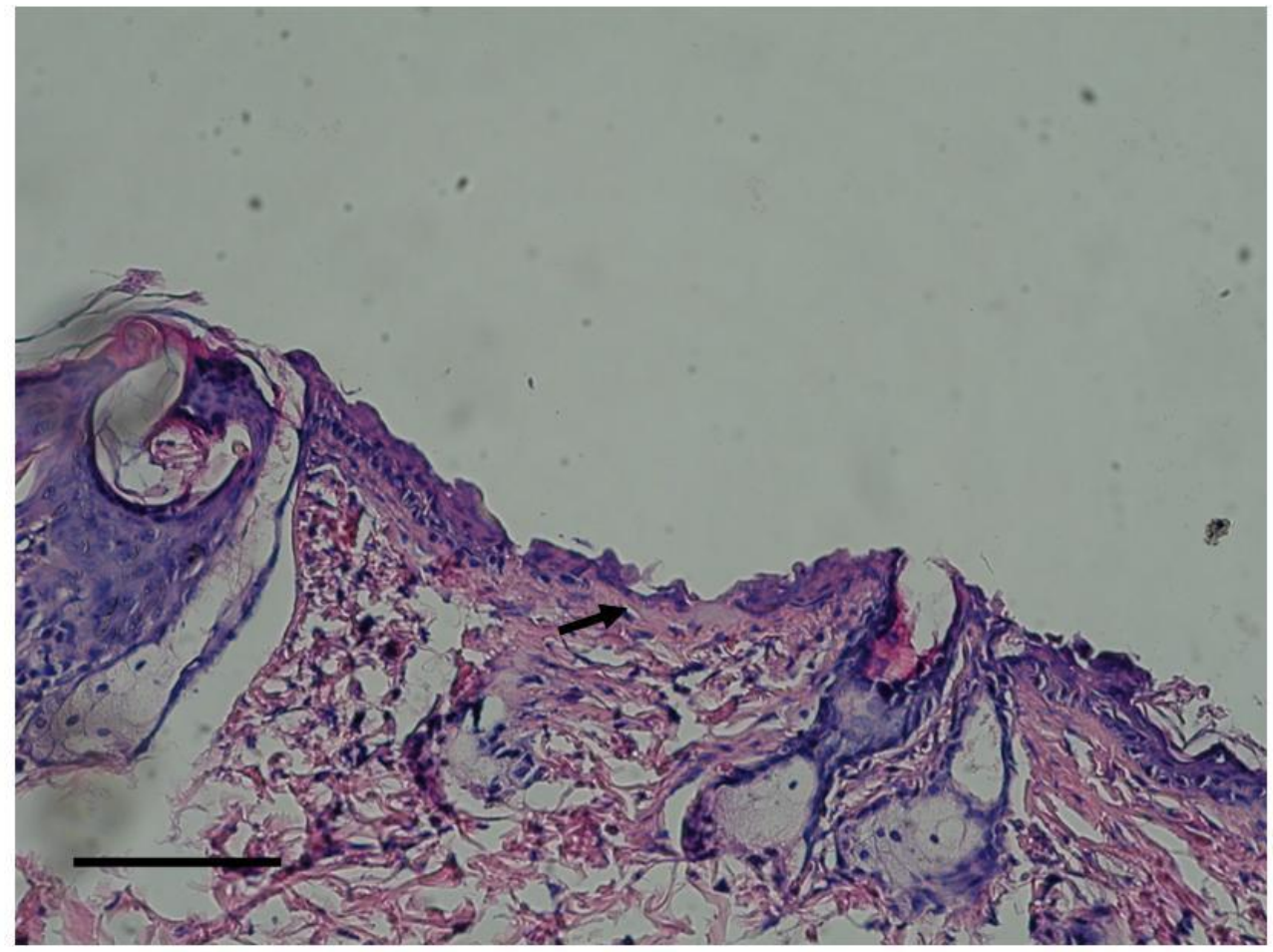

Figure 4. H\&E stain of nude mouse skin after Erbium:YAG laser at fluence of $13 \mathrm{~J} / \mathrm{cm}^{2}$ Part of epidermis was ablated. Dermal collagen was coagulated and appeared as eosinophilic degeneration (arrow). Bar $=150 \mu \mathrm{m}$

To further determine thermal effects of laser on dermis. We quantified the SHG signals of dermis at each specimen. The mean values of SHG of untreated dermis, dermis irradiated with $1.5 \mathrm{~J} / \mathrm{cm}^{2}$ and $13 \mathrm{~J} / \mathrm{cm}^{2}$ laser fluence were 24.49 , 5.22, and 0.77 respectively. The result reflected the fact that the structure lacking inversion symmetry responsible for the collagen SHG signal has been disrupted after laser irradiation. The higher fluence would resulted in more SHG signals decrease, indicating that laser at higher fluence produced more thermal damage. This is consistent with the findings of our previous study of thermally induced structural transitions of collagen fibers using second-harmonic generation microscopy ${ }^{22}$.

\section{Discussion}

We demonstrated the potential of multiphoton microscopy in evaluating Erbium:YAG laser-tissue interaction in this study. The epidermis ablated by Er:YAG laser appeared to be amorphous and increased AF. The residual non-ablated epidermis were visible and the thickness of residual epidermis could be measured in vivo. The thermal effects of Er:YAG laser were largely studied. ${ }^{14,26-28}$ After laser ablation of tissue, the residual thermal zone can induce collagen 
neogenesis, ${ }^{29}$ but too much thermal damage may result in unwanted complications. ${ }^{28}$ The evaluation of residual thermal zone depends on histologic examination previously, but the procedure is not real-time and invasive in nature, rendering biopsy patients after laser treatment for cosmetic purpose impossible. The findings of this study support the fact that AF and SHG microscopy is effective in identifying the extent of residual thermal damage after laser irradiation. The residual thermal damage of laser irradiation may induce increase of autofluorescence of tissue. Fig 3 showed amorphous enhanced AF mass at the residual keratinocyte debris after ablation of laser. Thermal denaturation results in the loss of collagen molecular organization which can have profounds influence on SHG. ${ }^{30}$ Consistent with our previous reports, ${ }^{21,22}$ SHG intensity decrease at high temperatures and is a useful predictor of dermal thermal damage. The importance of long-term monitoring to evaluate the microvascular response to light-based therapy has been proposed. ${ }^{31}$ The value of multiphoton microscopy in evaluating laser-tissue reaction in vivo awaits further investigation, and applying the technique as a real-time monitoring system during laser treatment is promising.

\section{Acknowledgement}

Support for this work is provided by the Far Eastern Memorial Hospital and National Research Program for Genomic Medicine of the National Science Council, Taiwan. Address correspondence to Dr. Woan-Ruoh Lee at

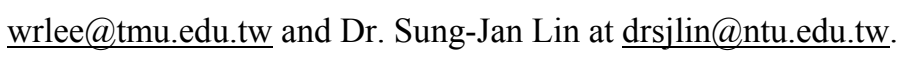

\section{Reference}

1. Jimenez, G. \& Spencer, J.M. Erbium:YAG laser resurfacing of the hands, arms, and neck. Dermatol Surg 25, 831-834; discussion 834-835 (1999).

2. Ostertag, J.U. et al. Congenital naevi treated with erbium:YAG laser (Derma K) resurfacing in neonates: Clinical results and review of the literature. Br J Dermatol 154, 889-895 (2006).

3. Orenstein, A. et al. Treatment of rhinophyma with Er:YAG laser. Lasers Surg Med 29, 230-235 (2001).

4. Lee, W.R., Shen, S.C., Lai, H.H., Hu, C.H. \& Fang, J.Y. Transdermal drug delivery enhanced and controlled by erbium:YAG laser: a comparative study of lipophilic and hydrophilic drugs. $J$ Controlled Release 75, 155-166 (2001).

5. Lee, W.-R. et al. Erbium:YAG laser-mediated oligonucleotide and DNA delivery via the skin: an animal study. $J$ Controlled Release 115, 344-353 (2006).

6. $\quad$ Shen, S.-C., Lee, W.-R., Fang, Y.-P., Hu, C.-H. \& Fang, J.-Y. In vitro percutaneous absorption and in vivo protoporphyrin IX accumulation in skin and tumors after topical 5-aminolevulinic acid application with enhancement using an erbium:YAG laser. J Pharm Sci 95, 929-938 (2006).

7. Lee, W.-R., Shen, S.-C., Wang, K.-H., Hu, C.-H. \& Fang, J.-Y. The effect of laser treatment on skin to enhance and control transdermal delivery of 5-fluorouracil. J Pharm Sci 91, 1613-1626 (2002).

8. $\quad$ Fang, J.Y., Lee, W.R., Shen, S.C., Fang, Y.P. \& Hu, C.H. Enhancement of topical 5-aminolaevulinic acid delivery by erbium:YAG laser and microdermabrasion: a comparison with iontophoresis and electroporation. $\mathrm{Br}$ 
J Dermatol 151, 132-140 (2004).

9. Fang, J.-Y. et al. Transdermal delivery of macromolecules by erbium:YAG laser. $J$ Controlled Release 100, 75-85 (2004).

10. Lee, W.-R., Shen, S.-C., Kuo-Hsien, W., Hu, C.-H. \& Fang, J.-Y. Lasers and microdermabrasion enhance and control topical delivery of vitamin C. J Invest Dermatol 121, 1118-1125 (2003).

11. Moody, B.R., McCarthy, J.E. \& Hruza, G.J. Collagen remodeling after 585-nm pulsed dye laser irradiation: an ultrasonographic analysis. Dermatol Surg 29, 997-999; discussion 999-1000 (2003).

12. Torkian, B.A. et al. Noninvasive measurement of ablation crater size and thermal injury after $\mathrm{CO} 2$ laser in the vocal cord with optical coherence tomography. Otolaryngol Head Neck Surg 134, 86-91 (2006).

13. Alster, T.S. Clinical and histologic evaluation of six erbium:YAG lasers for cutaneous resurfacing. Lasers Surg Med 24, 87-92 (1999).

14. Ross, E.V. et al. Use of a novel erbium laser in a Yucatan minipig: a study of residual thermal damage, ablation, and wound healing as a function of pulse duration. Lasers Surg Med 30, 93-100 (2002).

15. Rajadhyaksha, M., Grossman, M., Esterowitz, D., Webb, R.H. \& Anderson, R.R. In vivo confocal scanning laser microscopy of human skin: melanin provides strong contrast. Journal of Investigative Dermatology 104, 946-952 (1995).

16. Horn, M. et al. The use of confocal laser-scanning microscopy in microsurgery for invasive squamous cell carcinoma. Br J Dermatol 156, 81-84 (2007).

17. Denk, W., Strickler, J.H. \& Webb, W.W. Two-photon laser scanning fluorescence microscopy. Science 248, 73-76 (1990).

18. So, P.T., Dong, C.Y., Masters, B.R. \& Berland, K.M. Two-photon excitation fluorescence microscopy. Annual Review of Biomedical Engineering 2, 399-429 (2000).

19. Masters, B.R., So, P.T. \& Gratton, E. Multiphoton excitation microscopy of in vivo human skin. Functional and morphological optical biopsy based on three-dimensional imaging, lifetime measurements and fluorescence spectroscopy. Annals of the New York Academy of Sciences 838, 58-67 (1998).

20. Campagnola, P.J. \& Loew, L.M. Second-harmonic imaging microscopy for visualizing biomolecular arrays in cells, tissues and organisms. Nature Biotechnology 21, 1356-1360 (2003).

21. Lin, M.-G. et al. Evaluation of dermal thermal damage by multiphoton autofluorescence and second-harmonic-generation microscopy. Journal of Biomedical Optics 11, 064006 (2006).

22. Lin, S.-J. et al. Monitoring the thermally induced structural transitions of collagen by use of second-harmonic generation microscopy. Optics Letters 30, 622-624 (2005).

23. Sun, Y. et al. Investigating mechanisms of collagen thermal denaturation by high resolution second-harmonic generation imaging. Biophysical Journal 91, 2620-2625 (2006).

24. Lin, S.-J. et al. Evaluating cutaneous photoaging by use of multiphoton fluorescence and second-harmonic generation microscopy. Optics Letters 30, 2275-2277 (2005). 
25. Lin, S.-J. et al. Discrimination of basal cell carcinoma from normal dermal stroma by quantitative multiphoton imaging. Optics Letters 31, 2756-2758 (2006).

26. Majaron, B., Srinivas, S.M., Huang, H.e. \& Nelson, J.S. Deep coagulation of dermal collagen with repetitive Er:YAG laser irradiation. Lasers in Surgery \& Medicine 26, 215-222 (2000).

27. Khatri, K.A. The effects of variable pulse width of Er:YAG laser on facial skin. Dermatologic Surgery 27, 332-334 (2001).

28. Trelles, M.A., Mordon, S., Benitez, V. \& Levy, J.L. Er:YAG laser resurfacing using combined ablation and coagulation modes. Dermatologic Surgery 27, 727-734 (2001).

29. Kuo, T., Speyer, M.T., Ries, W.R. \& Reinisch, L. Collagen thermal damage and collagen synthesis after cutaneous laser resurfacing. Lasers in Surgery \& Medicine 23, 66-71 (1998).

30. Yeh, A.T., Choi, B., Nelson, J.S. \& Tromberg, B.J. Reversible dissociation of collagen in tissues. J Invest Dermatol 121, 1332-1335 (2003).

31. Choi, B., Jia, W., Channual, J., Kelly, K.M. \& Lotfi, J. The Importance of Long-Term Monitoring to Evaluate the Microvascular Response to Light-Based Therapies. J Invest Dermatol (2007). 